\title{
Modulation of LPS-induced nitric oxide production in intestinal cells by hydroxytyrosol and tyrosol metabolites: insight into the mechanism of action
}

Gabriele Serreli ${ }^{1}$, Maria Paola Melis ${ }^{1}$, Giulia Corona ${ }^{2}$ and Monica Deiana ${ }^{1, *}$

${ }^{1}$ Department of Biomedical Sciences, Unit of Experimental Pathology, University of Cagliari, Cittadella Universitaria SS 554, 09042 Monserrato (CA), Italy

${ }^{2}$ Health Sciences Research Centre, Life Sciences Department, Whitelands College, University of Roehampton, Holybourne Avenue, London, SW15 4JD, UK

* Corresponding author: Dr. Monica Deiana, Department of Biomedical Sciences, Unit of Experimental Pathology, University of Cagliari, Cittadella Universitaria SS 554, 09042 Monserrato (CA), Italy; Telephone: +39 070 6754126; Fax: +39 070 6754032; e-mail: mdeiana@ unica.it Gabriele Serreli, gabrieleserreli@hotmail.it Maria Paola Melis, mpmelis@unica.it Giulia Corona, giulia.corona@ roehampton.ac.uk

\begin{abstract}
Abbreviations: Akt, serine/threonine protein kinase; Caco-2, Caucasian colon adenocarcinoma; ERK, extracellular signal-regulated kinases; HT, hydroxytyrosol; HT sulf, hydroxytyrosol 3sulfate; HT glu, hydroxytyrosol-3-glucuronide; IBD, intestinal bowel diseases; I $\kappa$, inhibitor of kappa B; iNOS, inducible nitric oxide synthase; IKK, IкB kinase; JNK, c-Jun N-terminal kinases; LPS, lipopolysaccharide; MAPK, mitogen activated protein kinase; NF- $\kappa \mathrm{B}$, nuclear factor kappalight-chain-enhancer of activated B cells; NO, nitric oxide; Tyr, tyrosol; Tyr sulf, tyrosol sulfate; Tyr glu, tyrosol glucuronide.
\end{abstract}

Keywords: Caco-2 cells; lipopolysaccharide; metabolites; nitric oxide; olive oil 


\begin{abstract}
At intestinal level, after acute or chronic exposure to iNOS-derived NO a toxic mechanism of action leads to inflammation and degenerative diseases. The aim of this study was to investigate the effect of glucuronide and sulfate metabolites of the extra virgin olive oil phenols tyrosol (Tyr) and hydroxytyrosol (HT), in comparison with their parent compounds, on the release of NO following exposure to a pro-inflammatory stimulus, the bacterial lipopolysaccharide (LPS). Human colon adenocarcinoma cells (Caco-2), differentiated as normal enterocytes, were treated with pathological concentrations of LPS, in order to stimulate iNOS pathway, which involve NF-KB activation through IKB $\alpha$ phosphorylation and subsequent degradation induced by Akt or MAPKs. All the tested metabolites inhibited NO release induced by LPS, acting as inhibitors of iNOS expression, with an efficacy comparable to that of the parent compounds. HT and Tyr metabolites were effective in the inhibition of $\mathrm{IKB}_{\alpha} \alpha$ degradation. No one of the compounds was able to inhibit Akt activation, whereas they modulated p38 and ERK1/2 MAPK. Obtained data show that HT and Tyr metabolites are able to prevent a pathological NO overproduction at intestinal level, where they concentrate, thus significantly contributing to the protective activity exerted by their parent compounds against inflammation.
\end{abstract}




\section{Introduction}

Nitric oxide (NO) is a free radical compound which plays an important cellular signaling role within the mammalian body, where it is involved in many physiological and pathological processes (Habib and Ali, 2011; He et al., 2014). In the intestinal epithelium, NO production is strictly linked to the activation of the inducible nitric oxide synthase (iNOS) (Serra et al., 2018). At this level, NO may play, at low concentrations, a protective role against the inflammatory process, while after acute or chronic exposure a toxic mechanism of action leads to injurious effects. Indeed, its chronic release at high concentrations, due to an up-regulation of iNOS, have been correlated with the pathogenesis of inflammatory bowel diseases (IBD) (Marion et al., 2003) and colon cancer (Rao, 2004), mainly through the generation of peroxynitrite (Soufli et al., 2016). Several dietary phytochemicals have already been studied for their ability to act as anti-inflammatory agents, limiting NO abnormal release in intestinal cells through the inhibition of iNOS expression (Panaro et al., 2012; Romier-Crouzet et al., 2009; Somchit et al., 2014). The nuclear factor kappa-lightchain-enhancer of activated B cells $(\mathrm{NF}-\mathrm{\kappa} B)$ is a central target for the induction of iNOS expression by pro-inflammatory agents such as lipopolysaccharide (LPS) and stimulatory cytokines like interferon- $\gamma($ IFN- $\gamma$ ), interleukin-1 $\beta$ (IL-1 $\beta$ ), interleukin-6 (IL-6), and tumor necrosis factor- $\alpha$ (TNFa) (Abbas et al., 2016; Kleinert et al., 2003). This nuclear factor is usually activated after phosphorylation and subsequent degradation of its inhibitor $\mathrm{I} \kappa \mathrm{B}$, which is present in three different isoforms, I $\kappa \mathrm{B} \alpha, \mathrm{I} \kappa \mathrm{B} \beta$, and $\mathrm{I} \kappa \mathrm{B} \varepsilon$ (Kanarek et al., 2010). The extent of I $\kappa \mathrm{B}$ degradation depends on

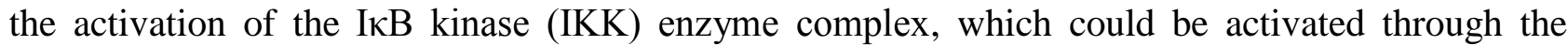
PI3/Akt (serine/threonine protein kinase) pathway (Kane et al., 1999). Akt can also regulate signaling pathways that lead to the induction of the NF-KB family of transcription factors, and it is specific for NF-KB, as other inducible transcription factors such as the Nuclear factor of activated T cells (NFAT) and the Activator protein 1 (AP-1) are not affected by Akt overexpression (Kane et 
al., 1999). IкB phosphorylation could also be led by activating key protein kinases such as mitogen activated protein kinases (MAPKs) p38, JNK, and ERK1/2 (Serra et al., 2018).

Phenolic compounds present in extra virgin olive oil, have been demonstrated to exert many potentially beneficial biological effects, starting at intestinal level (Deiana et al., 2018); in this site some of them, namely hydroxytyrosol (HT) and tyrosol (Tyr), may reach quite high concentrations, in the high $\mu \mathrm{M}$ range (Scalbert and Williamson, 2000).

Indeed, the bioavailability of HT and Tyr is poor due to an extensive metabolism (RodriguezMorato et al., 2016). Miro-Casas et al. (2003) showed that sulfated and glucuronidated HT and Tyr were the predominant metabolites found in human plasma and urine, and they have also been shown to concentrate in the intestinal lumen. Indeed, the content of sulfate and glucuronide metabolites in intestinal cells is relevant, due to intensive glucuronidation and sulfation which are major pathways of phase II xenobiotic metabolism in the human intestine (Rubiò et al., 2014a). Thus, at this location major metabolites might significantly contribute to the protective action ascribed to their parent compounds, acting as antioxidants and anti-inflammatory agents (Serreli et al., 2018). Nevertheless, recent studies pointed out that sulfate and glucuronide moieties can be deconjugated once they get intracellular environment, thus they may act also as free parent compounds (Catalan et al., 2015; Rubiò et al., 2014b).

HT and Tyr have been widely studied for their numerous beneficial effects against cardiovascular (Bulotta et al., 2014; Hu et al., 2014; Muriana et al., 2017), neuronal (Hu et al., 2014), intestinal (Deiana et al., 2018) and neoplastic diseases (Casaburi et al., 2013). These compounds are well known for their direct antioxidant capacity (Bulotta et al., 2014; Rodriguez-Morato et al., 2016) but they are also able to modulate molecular pathways acting as indirect antioxidant, anti-inflammatory (Cicerale et al., 2012; Santangelo et al., 2018), anti-proliferative (Corona et al., 2009) and anticancer agents (Guichard et al., 2006).

In previous studies, HT proved to be effective in the downregulation of kinases such as MAPKs, ERK1/2 and JNK, and Akt, counteracting $\mathrm{H}_{2} \mathrm{O}_{2}$-induced injury in enterocytes and inhibiting the 
proliferation of human colon adenocarcinoma cells (Corona et al., 2009). HT and Tyr have also been found to reduce pro-inflammatory cytokines secretion in peripheral blood mononuclear cells by modulating p38 and JNK kinases (Serra et al., 2017). The activation of these kinases in response to pro-inflammatory stimuli (namely TNF- $\alpha$, IFN- $\gamma$, IL-6, and IL-1 $\alpha$ ), and bacterial component LPS is known to be involved in the expression of iNOS, responsible for the increased NO production at the site of inflammation (Murakami, 2009; Viatour et al., 2005).

The goal of this study was to evaluate the ability of HT and Tyr sulfate and glucuronide metabolites, in comparison with their parent compounds, to act as anti-inflammatory agents through the inhibition of NO over-release in human colon adenocarcinoma cells (Caco-2) differentiated as normal enterocytes, following treatment with pathological concentrations of LPS. In particular, we focused our attention on the mechanisms of their modulatory action on iNOS expression, investigating their ability to modulate Akt signaling pathway or MAPKs (JNK, p38 and ERK1/2) in relation to NF- $\mathrm{NB}$ activation. 


\section{Materials and methods}

\subsection{Chemicals}

LPS from Escherichia coli, Tyr (2-(4-Hydroxyphenyl)ethanol, purity 98\%, cod. 188255, CAS Number 501-94-0), HT (2-(3,4-Dihydroxyphenyl)ethanol, purity 98\%, cod. H4291, CAS Number: 10597-60-1), L-arginine, sodium nitrite, Griess' reagent, Bradford reagent, Phosphatase Inhibitor Cocktail II, Protease Inhibitor Cocktail and Thiazolyl Blue Tetrazolium Bromide (MTT) were purchased from Sigma Aldrich (Milan, Italy). Tyr sulf (p-Hydroxyphenethyl Alcohol p-(Hydrogen Sulfate) Sodium Salt; 4-(Sulfooxy)benzeneethanol Sodium Salt, purity 98\%, cod. T947815, CAS Number: not available), HT sulf (4-(2-Hydroxyethyl)-1,2-benzenediol 2-(Hydrogen Sulfate) Sodium, purity 98\%, cod. H977010, CAS Number: 1391053-88-5), HT glu (2-Hydroxy-5-(2hydroxyethyl)phenyl $\beta$-D-Glucopyranosiduronic Acid, purity 97\%, cod. H977000, CAS Number: 425408-50-0), and Tyr glu (4-(2-Hydroxyethyl)phenyl $\beta$-D-Glucopyranosiduronic Acid, purity 98\%, cod. T947810, CAS number: 28116-28-1) were purchased from Toronto Research Chemicals (North York, ON, Canada). Cell culture materials were purchased from Invitrogen (Milan, Italy). Phenolic compounds were dissolved in $\mathrm{MeOH}$ (stock solution $1 \mathrm{mg} / \mathrm{mL}$ ) and stored at $-20^{\circ} \mathrm{C}$. LPS was freshly prepared as a water solution $(1 \mathrm{mg} / \mathrm{mL})$ just before treatments.

\subsection{Cell cultures}

Caco-2 cells (ECACC Salisbury, Wiltshire UK) were cultured in Dulbecco's modified Eagle's medium (DMEM), supplemented with 10\% heat-inactivated bovine serum, $2 \mathrm{mM}$ L-glutamine, $1 \%$ non-essential amino acids, $100 \mathrm{U} / \mathrm{ml}$ penicillin, and $100 \mathrm{mg} / \mathrm{ml}$ streptomycin, in monolayers at $37^{\circ} \mathrm{C}$ in a humidified atmosphere of $5 \% \mathrm{CO}_{2}$ (Incani et al., 2016), replacing the medium twice a week. For experimental studies Caco-2 cells, at passage 45-60, were plated and used 18-21 days post seeding, when fully differentiated.

\subsection{MTT viability test}


The MTT assay was assessed on Caco-2 cells (Rosa et al., 2011) in order to evaluate any toxic activity of the tested compounds. Cells were seeded in 96-well plates $\left(5 \times 10^{4}\right.$ viable cells $/ \mathrm{mL}$ in 100 $\mu \mathrm{L}$ ), exposed to various concentrations of the compounds alone (HT, Tyr, Tyr sulfate, HT sulfate, HT glucuronide and Tyr glucuronide at $0.1,1$ and $10 \mu \mathrm{M}$, in serum free medium) or to the same phenolic compounds preincubated ( $1 \mu \mathrm{M}, 30 \mathrm{~min}$ ) prior to LPS co-exposure (LPS $1 \mu \mathrm{g} / \mathrm{mL})$ or an equivalent volume of $\mathrm{MeOH}$ (vehicle of all the compounds) for the controls, and incubated for $72 \mathrm{~h}$. After incubation, the medium was removed and $100 \mu \mathrm{L}$ of MTT solution $(5 \mathrm{mg} / \mathrm{mL}$ of fresh medium) was added and left for $6 \mathrm{~h}$ at $37^{\circ} \mathrm{C}$. The medium was then aspirated, $100 \mu \mathrm{L}$ of dimethyl sulfoxide (DMSO) were added in each well and the absorbance was read at $570 \mathrm{~nm}$ by using a micro plate reader (Infinite 200, Tecan, Salzburg, Austria).

\subsection{Measure of nitric oxide}

In order to evaluate NO production, Caco- 2 cells were cultured in 6-well plates $\left(5 \times 10^{4}\right.$ cells $/ \mathrm{mL}$ in 2 $\mathrm{mL}$ ) in phenol red-free DMEM with L-arginine $0.1 \mathrm{mM}$ and $2.5 \%$ fetal bovine serum. A time course analysis of NO release was performed by incubating cells from 0 to $72 \mathrm{~h}$ after treatments with LPS $1 \mu \mathrm{g} / \mathrm{mL}$. In another set of experiments, cells were pretreated with the phenolic compounds tested in this study (HT, Tyr, Tyr sulfate, HT sulfate, HT glucuronide and Tyr glucuronide at $1 \mu \mathrm{M}$ for $30 \mathrm{~min})$ prior to LPS co-exposure $(1 \mu \mathrm{g} / \mathrm{mL})$ for $48 \mathrm{~h}$. After incubation, the medium was collected from the wells and the nitrite concentration was determined by mixing 100 $\mu \mathrm{L}$ of the same medium with an equal volume of Griess' reagent and incubating for 20 min at room temperature. Then, plate was read at $540 \mathrm{~nm}$, and nitrite levels were determined with a sodium nitrite standard curve ranging from 0.1 to $10 \mu \mathrm{M}$. The results were expressed as $\mu \mathrm{M}$ of nitrites secreted by the cells.

\subsection{Western blot analyses}


To assess western blot analyses, Caco- 2 cells were seeded in 6 -well plates $\left(5 \times 10^{4}\right.$ cells $/ \mathrm{mL}$ in $\left.2 \mathrm{~mL}\right)$ in DMEM with L-arginine $0.1 \mathrm{mM}$ and $2.5 \%$ fetal bovine serum. Once differentiated, cells were pretreated with the phenolic compounds (HT, Tyr, Tyr sulfate, HT sulfate, HT glucuronide and Tyr glucuronide at $1 \mu \mathrm{M}$ for $30 \mathrm{~min})$ prior to LPS co-exposure $(1 \mu \mathrm{g} / \mathrm{mL})$ for 2 or $48 \mathrm{~h}$. Then, supernatant was removed and Caco-2 cells were lysed in $200 \mu \mathrm{L}$ of commercial lysis buffer CellLytic M (Sigma Aldrich, Milan, Italy) supplemented with Pierce ${ }^{\mathrm{TM}}$ phosphatase and protease inhibitor mini tablets (Thermo Fisher Scientific, Waltham, MA, USA) for protein extraction. Lysates were centrifuged $\left(15.000 \mathrm{x} \mathrm{g}, 10 \mathrm{~min}, 4^{\circ} \mathrm{C}\right)$, supernatants collected and used to determine total protein concentration by the Bradford assay (Bradford, 1976) or stored at $-20^{\circ} \mathrm{C}$ to be used for western blot assays. Twenty micrograms of reduced and denatured proteins were separated on $10 \%$ polyacrylamide gels and thereupon transferred into nitrocellulose membranes. Membranes were blocked with $25 \mathrm{~mL}$ of TTBS (tris-buffered saline with Tween 20, composed by Tris/HCl $20 \mathrm{mM}$, $\mathrm{pH} 7.5,100 \mathrm{mM} \mathrm{NaCl}$ and $0.1 \%$ Tween 20) 4\% dry milk for $30 \mathrm{~min}$ at room temperature. Primary poly-clonal antibodies, anti-IкB $\alpha$, anti-phospho IкB $\alpha$, anti-Akt, anti-phospho Akt, anti- $\beta$ actin, antiERK1/2, anti-phospho ERK1/2, anti-p38, anti-phospho p38, anti-JNK, anti-phospho JNK and antiiNOS antibodies, (Cell Signaling Technology, Inc., Danvers, Massachussets, USA) in TTBS 1\% dry milk (dilution 1:1000) were added to the membranes and kept overnight on a three-dimensional rocking table at $4^{\circ} \mathrm{C}$. Membranes were washed two times with TTBS before incubation with goat secondary anti-rabbit IgG conjugated to horseradish peroxidase (1:2000 dilution) (Sigma Aldrich, Milan, Italy) in TTBS $1 \%$ milk for $45 \mathrm{~min}$ at room temperature and then washed twice with TTBS and once with TBS (Tris/HCl $20 \mathrm{mM}$, pH 7.5 and $100 \mathrm{mM} \mathrm{NaCl}$ ). The bands were visualized by using the ChemiDoc ${ }^{\mathrm{TM}}$ XRS+ System (Bio-Rad Laboratories, Inc., Hercules, California, USA).

\subsection{Statistical Analyses}


One-way analysis of variance (ANOVA) followed by Tukey's test was performed in order to highlight possible significant differences between groups $(\mathrm{p}<0.05)$ using the GraphPad Prism 5 software (GraphPad software, San Diego, CA, USA). 


\section{Results}

\subsection{Evaluation of cell viability}

To investigate the potential protective effect of HT and Tyr sulfate and glucuronide metabolites, in comparison with their parent compounds, against LPS-induced pro-inflammatory effects in Caco-2 cell monolayers, control experiments were initially carried out to assess cell viability after treatment with the phenolic compounds, LPS alone or LPS in presence of the compounds. As reported in Figure 1, cell viability remained unchanged in presence of the tested phenolic compounds in the concentration range $0.1-10 \mu \mathrm{M}$ and in presence of the chosen concentration of LPS $(1 \mu \mathrm{g} / \mathrm{mL})$ alone or co-incubated with the phenolic compounds $(1 \mu \mathrm{M}$, pre-incubated 30 min prior to LPS coexposure).

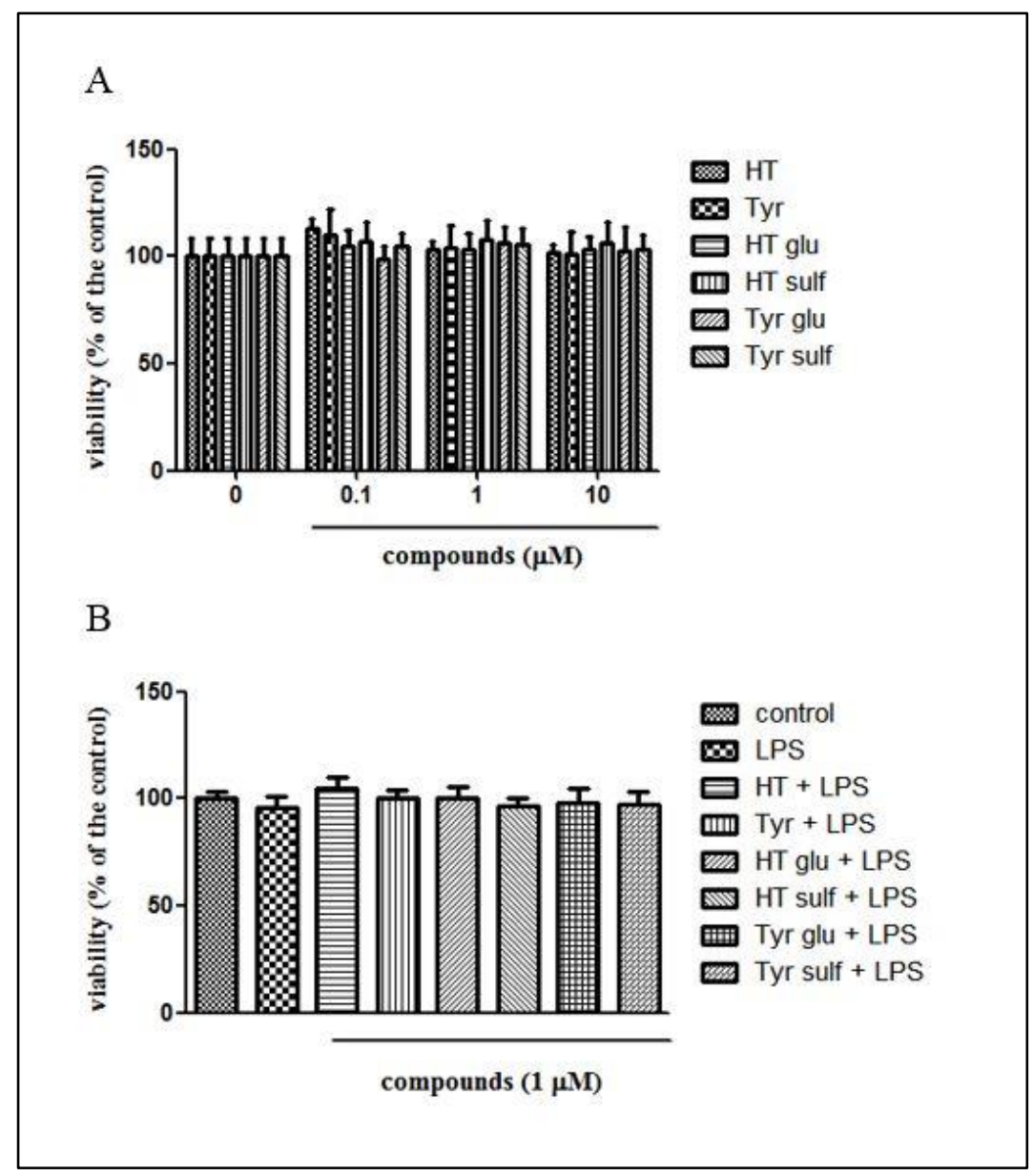

Fig.1. Percentage of cell viability compared to control (100\%) of Caco-2 cells incubated for $72 \mathrm{~h}$ with different concentrations of hydroxytyrosol (HT), tyrosol (Tyr) and their glucuronide (glu) and sulfate (sulf) metabolites (0.1-10 
$\mu \mathrm{M})(\mathrm{A})$ or incubated with LPS $(1 \mu \mathrm{g} / \mathrm{mL})$ alone or co-incubated with the phenolic compounds $(1 \mu \mathrm{M}$, pre-incubated 30 min prior to LPS co-exposure) (B). Each column represents the mean \pm SD of independent experiments $(n=12)$. 


\subsection{Effect on NO production and iNOS expression}

NO release in Caco-2 cells after treatment with LPS for 0-72 h was measured as nitrites content in culture medium. LPS was able to induce NO release, showing significant differences in nitrite levels compared to control starting from $48 \mathrm{~h}$ of incubation, where NO amount doubled for LPS treated samples (Figure 2).

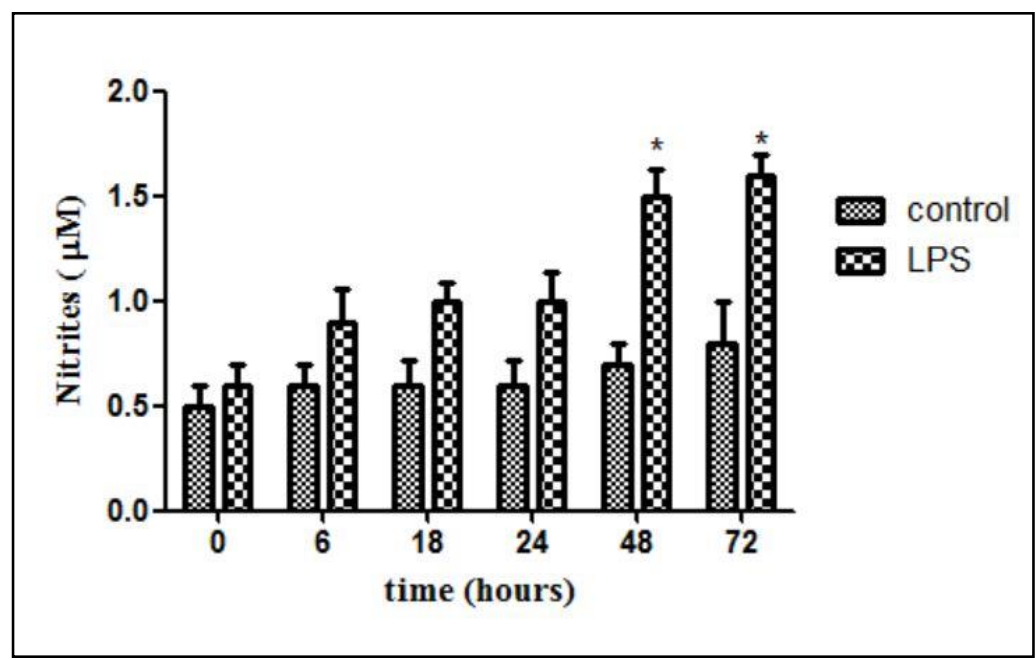

Fig.2. NO production (expressed as $\mu \mathrm{M}$ of nitrites) in Caco-2 cells untreated or treated with LPS $(1 \mu \mathrm{g} / \mathrm{mL}$ ) for 0-72 h. Measurements were assessed in supernatant by using Griess' reagent method. Each column represents the mean \pm SD of independent experiments $(n=16){ }^{*}=p<0.05$ LPS vs untreated cells at the same incubation time.

NO production in Caco-2 cells after 48 h LPS exposure with and without pretreatment (30 min prior to LPS co-exposure) with the phenolic compounds $(1 \mu \mathrm{M})$ was then analyzed. As shown in Figure 3, all the compounds significantly counteracted NO production. Among the tested compounds, HT and HT sulfate were the most effective in limiting the release of NO, which level was $54 \%$ and $57 \%$ respectively of the amount detected in LPS treated cells, near to that of control. 


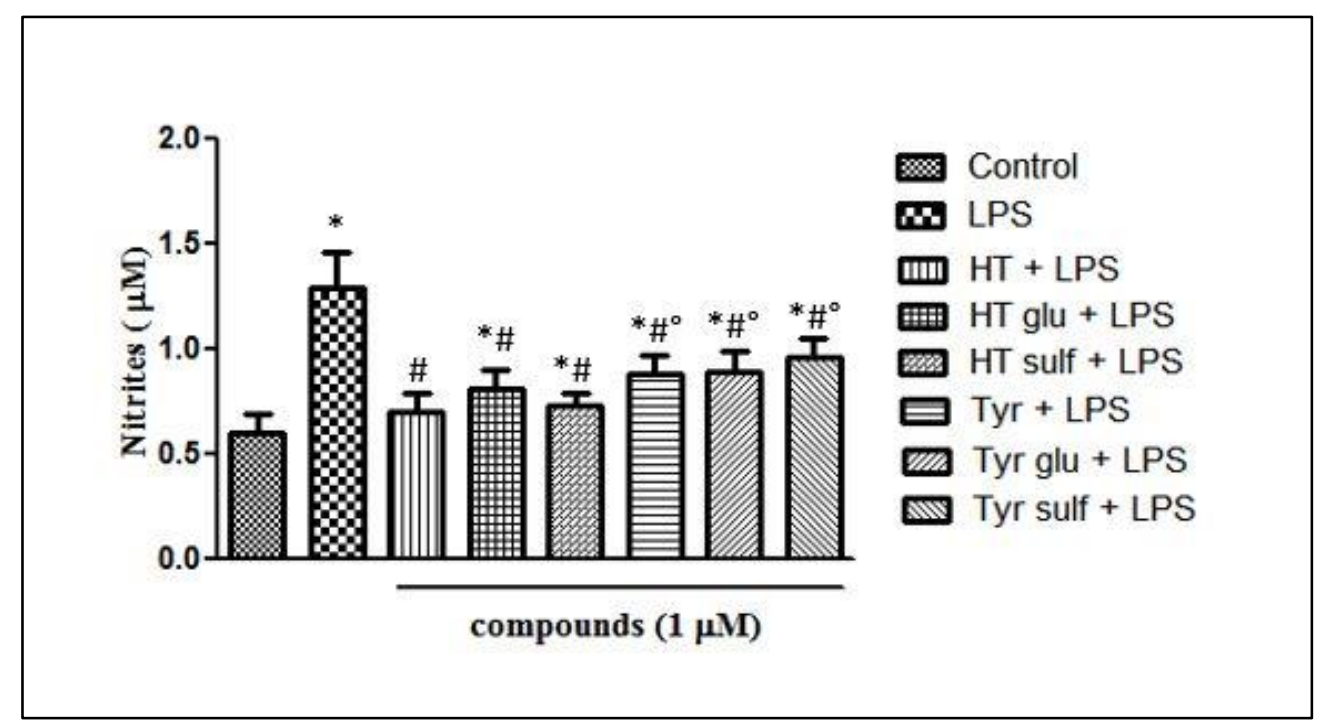

Fig.3. NO production (expressed as $\mu \mathrm{M}$ of nitrites) in Caco- 2 cells treated with LPS $(1 \mu \mathrm{g} / \mathrm{mL})$ for $48 \mathrm{~h}$ and pretreated (30 min) with hydroxytyrosol (HT), tyrosol (Tyr) and their glucuronide (glu) and sulfate (sulf) metabolites (1 $\mu \mathrm{M})$ prior to LPS co-exposure. Measurements were assessed in supernatant by using Griess' reagent method. Each column represents the mean \pm SD of independent experiments $(n=16)$. Significant differences among groups are reported using different superscript symbols $(\mathrm{p}<0.05) ; *=$ significant vs control; $\#=$ significant vs $\mathrm{LPS} ;{ }^{\circ}=$ significant vs HT + LPS and HT sulf + LPS.

A significant increase of iNOS expression in LPS treated cells with respect to the control was also observed in parallel to NO release. In Caco-2 cells pretreated with the phenolic compounds LPS induction of iNOS expression was significantly inhibited, though enzyme level was still higher than that of the control (Figure 4). All the metabolites exerted a significant effect $(\mathrm{p}<0.05)$ in limiting iNOS expression with respect to the LPS treated samples, comparable to that of the parent compounds. 


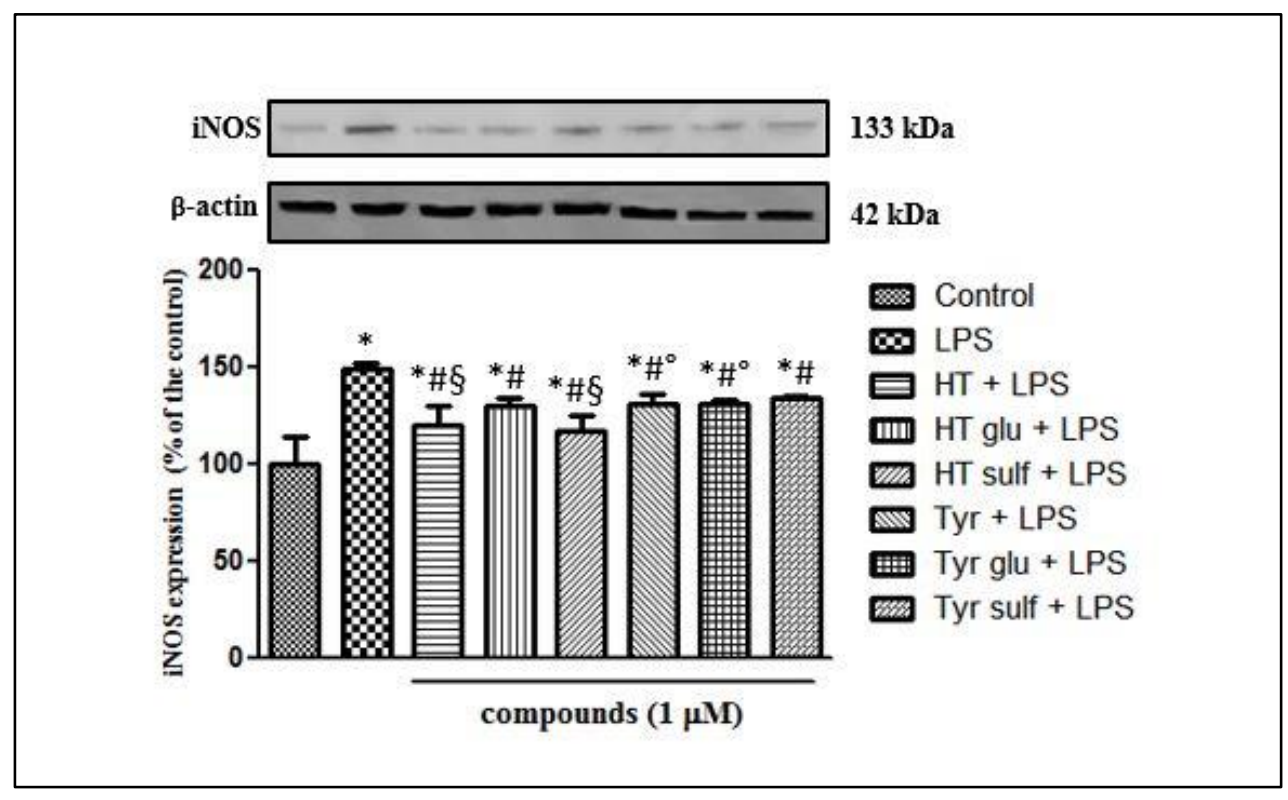

Fig.4. Expression of iNOS, reported as percentage of the control, in Caco-2 cells treated with LPS $(1 \mu \mathrm{g} / \mathrm{mL})$ for $48 \mathrm{~h}$ and pretreated (30 min) with hydroxytyrosol (HT), tyrosol (Tyr) and their glucuronide (glu) and sulfate (sulf) metabolites $(1 \mu \mathrm{M})$ prior to LPS co-exposure. Each column represents the mean \pm SD of independent experiments $(\mathrm{n}=6)$. Significant differences among groups are reported using different superscript symbols $(\mathrm{p}<0.05) ; *$ significant vs control; \# = significant vs LPS; ${ }^{\circ}=$ significant vs HT sulf + LPS; $\S=$ significant vs Tyr sulf + LPS. Representative WB image of the treatment is shown. $\beta$-actin detection was used as a loading control for each sample.

\subsection{Prevention of IKB $\alpha$ degradation}

IкB $\alpha$ phosphorylation by IKK is a main step which allows NF-кB to modulate iNOS expression. Figure 5 reports the results of IkB $\alpha$ modulation occurred in Caco- 2 cells after LPS treatment for 48 h. A western blot analysis was assessed to evaluate the ratio between $\operatorname{IkB} \alpha$ and its phosphorylated form. ІкВ $\alpha$ phosphorylation was predominant in cells treated only with LPS, where phospho ІкB $\alpha /$ total ІкВ $\alpha$ ratio was four times greater than that of the control, while pretreatment with HT and its sulfate and glucuronide metabolites and to a lesser extent with Tyr and metabolites significantly inhibited IKB $\alpha$ phosphorylation, thus blocking its degradation. 


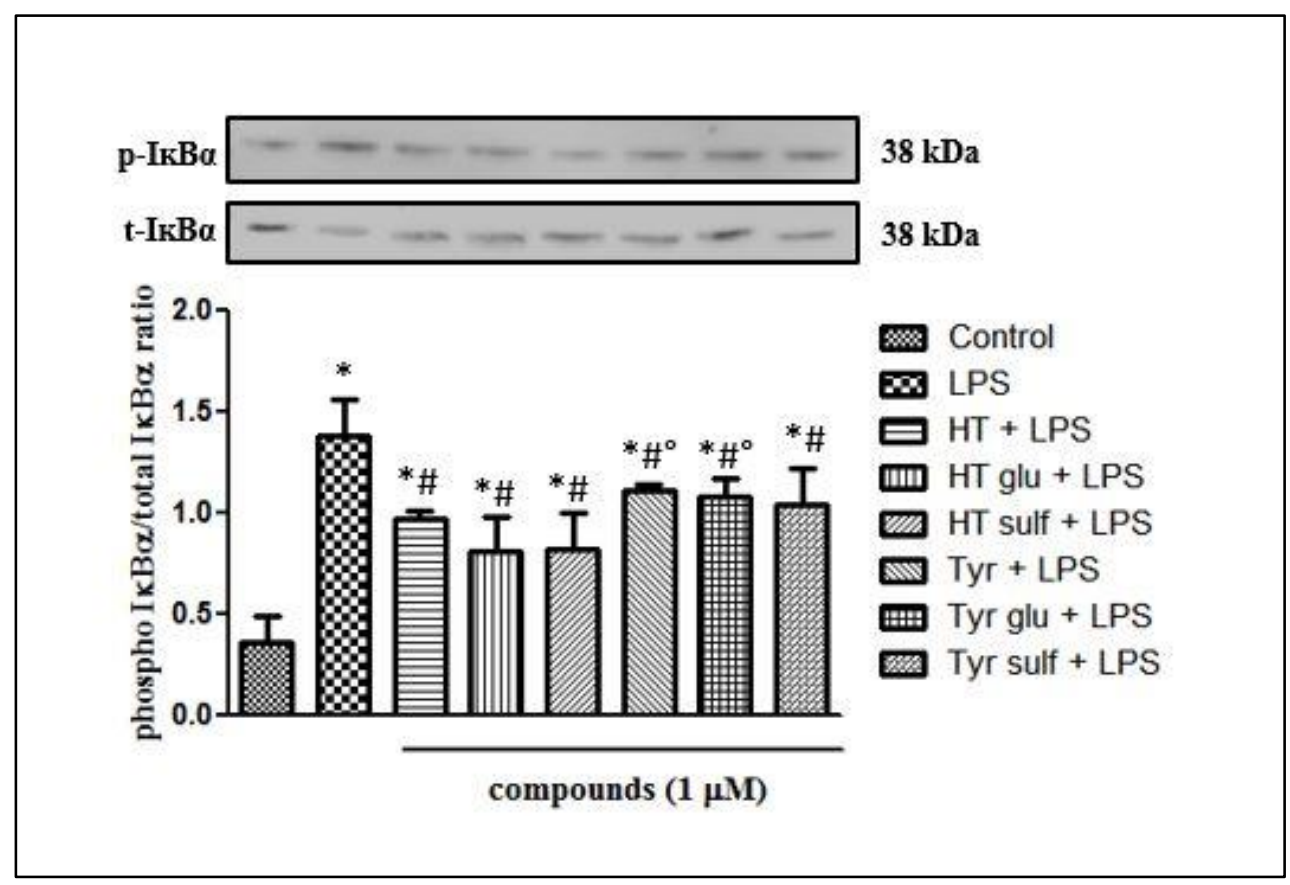

Fig.5. Degradation of IкB $\alpha$ reported as phospho IкB $\alpha /$ total IкB $\alpha$ ratio in Caco-2 cells treated with LPS (1 $\mu \mathrm{g} / \mathrm{mL})$ for 48 h and pretreated (30 min) with hydroxytyrosol (HT), tyrosol (Tyr) and their glucuronide (glu) and sulfate (sulf) metabolites $(1 \mu \mathrm{M})$ prior to LPS co-exposure. Each column represents the mean $\pm \mathrm{SD}$ of independent experiments $(\mathrm{n}=6)$. Significant differences among groups are reported using different superscript symbols $(\mathrm{p}<0.05) ; *=$ significant vs control; \# = significant vs LPS ${ }^{\circ}=$ significant vs HT glu + LPS and HT sulf + LPS. Representative WB image of the treatment is shown.

\subsection{Modulation of Akt and MAPK p38, JNK and ERK1/2}

The ability of the phenolic compounds to modulate LPS stimulation of intracellular signaling pathways involved in NO release was evaluated, focusing in particular on the Akt pathway and on MAPK JNK, p38 and ERK 1/2. As shown in Figure 6 and 7, incubation with LPS led to a significant change in Akt kinase, p38 and ERK1/2 phosphorylation state already after $2 \mathrm{~h}$, as assessed by western blot analysis. The extent of Akt and ERK phosphorylation in cells treated only with LPS was three times, and twice in the case of p38, the one detected in the control, whereas JNK phosphorylation was not observed. None of the tested metabolites nor their parent compounds was able to modulate Akt phosphorylation, as phospho Akt/total Akt ratio was the same in all LPS 
treated samples, irrespective to the presence of the phenolic compounds (Figure 6); all of them instead limited LPS effect on p38 and ERK1/2 activation.

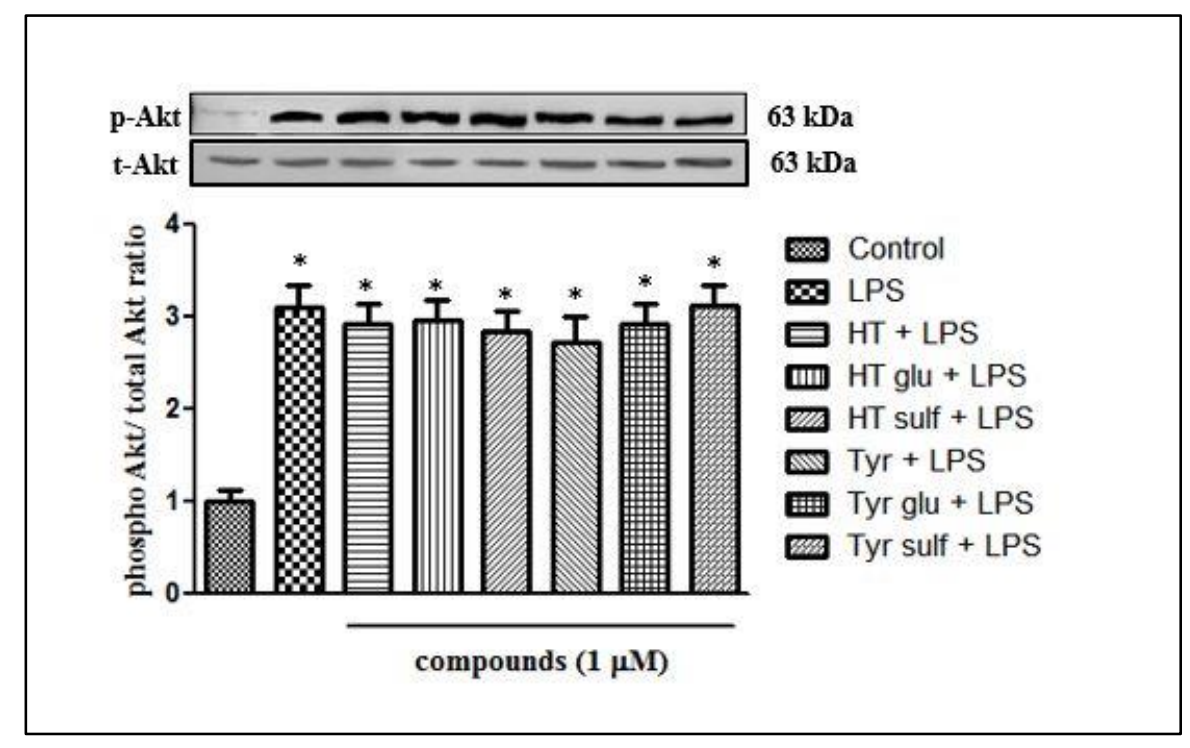

Fig.6. Activation of Akt reported as phospho Akt/total Akt ratio in Caco-2 cells treated with LPS $(1 \mu \mathrm{g} / \mathrm{mL})$ for $2 \mathrm{~h}$ and pretreated (30 min) with hydroxytyrosol (HT), tyrosol (Tyr) and their glucuronide (glu) and sulfate (sulf) metabolites (1 $\mu \mathrm{M})$ prior to LPS co-exposure. Each column represents the mean $\pm \mathrm{SD}$ of independent experiments $(\mathrm{n}=6)$. Significant differences among groups are reported using different superscript symbols $(\mathrm{p}<0.05) ; *=$ significant vs control. Representative WB image of the treatment is shown.

Pretreatment with both HT and Tyr metabolites and their parent compounds exerted a comparable efficacy. p38 and ERK1/2 phosphorylation was significantly inhibited with respect to the cells treated with only LPS $(\mathrm{p}<0.05)$, although the extent of the phosphorylation was still significantly above the control level (Figure 7). 


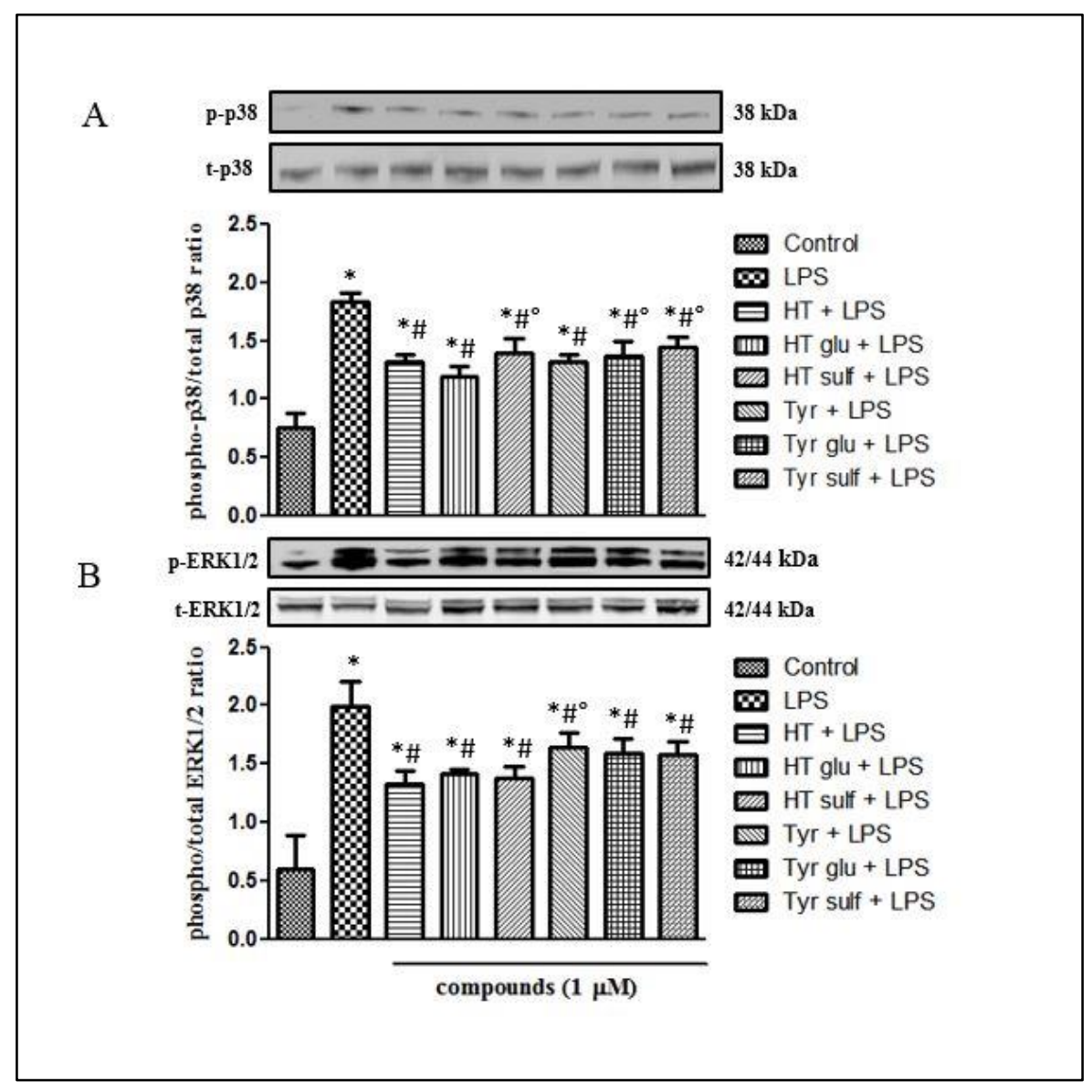

Fig.7. Modulation of MAPK p38 (A) and ERK1/2 (B) reported as phospho p38/total p38 and phospho ERK1/2/total ERK1/2 ratios in Caco-2 cells treated with LPS $(1 \mu \mathrm{g} / \mathrm{mL})$ for $2 \mathrm{~h}$ and pretreated (30 min) with hydroxytyrosol (HT), tyrosol (Tyr) and their glucuronide (glu) and sulfate (sulf) metabolites (1 $\mu \mathrm{M})$ ) prior to LPS co-exposure. Each column represents the mean \pm SD of independent experiments $(n=6)$. Significant differences among groups are reported using different superscript symbols $(\mathrm{p}<0.05) ;(\mathrm{A}) *=$ significant vs control; $\#=$ significant vs LPS ${ }^{\circ}=$ significant vs HT glu + LPS; $(\mathrm{B}) *=$ significant vs control; \# = significant vs LPS ${ }^{\circ}=$ significant vs HT + LPS. Representative WB image of the treatment is shown. 


\section{Discussion}

Olive oil polyphenols are some of the most studied phenols for their health properties (Bendini et al., 2007; De la Torre, 2008). Among them, HT and Tyr are highly concentrated right at intestinal level (Scalbert \& Williamson, 2000) and have been recognized to exert several health effects, acting not only as direct antioxidants but also influencing gene expression and intracellular signaling mechanisms (Serreli et al., 2018). However, the relevance of in vitro data for the interpretation of in vivo effects of these phenols is controversial, since the oral bioavailability of these compounds is rather limited due to their metabolization mainly to sulfates and glucuronides (Serreli et al., 2018). The present study aimed to explore for the first time the ability of HT and Tyr glucuronide and sulfate metabolites, in comparison to their parent compounds, to act as anti-inflammatory agents by the modulation of iNOS-derived NO production at intestinal level. Compounds were tested at a biologically relevant concentration, $1 \mu \mathrm{M}$, which could be found in circulation after dietary consumption (Pastor et al., 2016) as well as in the gut lumen, where they may reach even higher concentrations (Jenner et al., 2005).

As a model of human intestinal epithelium Caco-2 cell monolayers were used. These cells derive from a human colon adenocarcinoma; once in culture they undergo a process of spontaneous differentiation into normal mature enterocytes (Jochems et al., 2018). iNOS is not usually expressed in these cells, but its expression can be induced by bacterial LPS, cytokines, and other toxic or proinflammatory agents (Förstermann \& Sessa, 2012). Once expressed, iNOS is constantly active and not regulated by intracellular $\mathrm{Ca}^{2+}$ concentrations and produces large amounts of NO (Förstermann $\&$ Sessa, 2012). In order to activate the pathway which leads to NO production, Caco-2 cells were treated with the pro-inflammatory agent LPS, given at the pathological concentration of $1 \mu \mathrm{g} / \mathrm{mL}$ (Guo et al., 2015), which has already been used for this purpose in other studies (Panaro et al., 2012; Romier et al., 2008; Van de Walle et al., 2010). LPS, the major cell wall component of Gramnegative bacteria, is abundant in the gut lumen and it is correlated to the pathogenesis of IBD when it reaches elevated circulating levels (over $10 \mathrm{ng} / \mathrm{mL}$ ) (Guo et al., 2013). In Caco-2 cells LPS 
exposure led to a highly significant production of NO, whose level was doubled with respect to the control, as a consequence of iNOS activation. NO overproduction was prevented by all the tested compounds, that reduced the level detected in LPS treated cells, up to $50 \%$ in the case of HT and HT sulfate. This partial inhibition of NO production is biologically relevant, as the maintenance of intestinal homeostasis is associated with the release of low concentrations of NO, which can play a protective role (Chokshi et al., 2008). Conversely, an excessive and prolonged iNOS-derived NO can lead to inflammatory conditions that increase mucosal permeability, thus triggering IBD (Chen and Kitts, 2015).

The protective action of the tested phenolic compounds was due to their ability to inhibit iNOS expression in Caco-2 cells, as indicated by western blot analysis. iNOS down-regulation by HT, HT acetate and some other HT derivatives was demonstrated by Maiuri et al. (2005) and Aparicio-Soto et al. (2015) in two different macrophage cell lines. Other studies highlighted the capacity of polyphenols with different chemical structures to inhibit iNOS expression in Caco-2 cells: resveratrol, for instance, acted as iNOS inhibitor (Cosan et al., 2010), counteracting NF- $\kappa$ B activation through $\mathrm{IkB} \alpha$ degradation (Panaro et al., 2012). iNOS is usually over-expressed after activation of NF-kB (Serra et al., 2018), which is consequent to its translocation into the nucleus. This translocation is allowed when its inhibitor IKB $\alpha$ gets phosphorylated by its kinase IKK (Gupta et al., 2010) which, in turn, could be activated by phosphorylated Akt or other kinases (Hoesel and Schmidt, 2013). It was previously shown that polyphenols can modulate this pathway, influencing NF-кB-induced iNOS gene expression (Bognar et al., 2013; Panaro et al., 2012). Hence, we evaluated the ability of HT and Tyr metabolites and their parent compounds to inhibit iNOS expression through NF-кB modulation, acting on IкB $\alpha$. LPS treatment induced an increase of the phospho IкB $\alpha /$ total IKB $\alpha$ ratio, which was reverted in presence of the phenolic compounds. It is interesting to note that compounds which better inhibited NO production, HT and HT sulfate, were also the most effective, together with HT glucuronide, in blocking $\mathrm{IKB} \alpha$ phosphorylation and then NF-KB activation. A possible mechanism of the outstanding activity of these compounds in 
counteracting $\operatorname{IKB} \alpha$ degradation could be linked to the ability to inhibit LPS-induced Akt phosphorylation. Indeed, Caco-2 cells treated with LPS showed a significant increase in Akt phosphorylation, in accordance to Xiong et al. (2015). However, pretreatment with the tested phenolic compounds did not exert in our experimental conditions any effect on Akt phosphorylation, contrary to what demonstrated in hepatocellular carcinoma (Zhao et al., 2014), and neuronal cells (Fu and $\mathrm{Hu}, 2016)$ treated with HT. Beyond the Akt-induced IKK activation, LPS is known to induce IкB phosphorylation also through the activation of kinases such as MAPK p38, JNK, and ERK1/2, all of which are involved in activating key transcription factors, including NF$\mathrm{\kappa B}$ (Murakami et al., 2009). In support of the MAPKs down-regulation in the context of IкB $\alpha$ phosphorylation, Cardeno et al. (2014) proved in murine macrophages that olive oil phenolic extracts reduced LPS-induced oxidative stress and inflammatory responses, through the downregulation of iNOS, which was linked to a reduced MAPKs phosphorylation and nuclear NF-кB translocation. In this study, LPS treatment in Caco-2 cells induced phosphorylation of MAPK p38 and ERK1/2, while no significant change in the phospho/total ratio of JNK was observed. It is conceivable that the concentration of LPS used $(1 \mu \mathrm{g} / \mathrm{mL})$ was not adequate to stimulate JNK activation. In fact, JNK activation in Caco-2 cells, as well as p38 and ERK1/2, was observed by Sun et al. (2013) with concentration of LPS 10-fold higher. Overall, all the compounds tested in this study were effective in counteracting p38 and ERK1/2 LPS-induced activation. This finding is in agreement with other studies regarding olive oil phenolics and their capacity of down-regulating MAPKs activity in intestinal (Serra et al., 2018) endothelial (Catalan et al., 2018), peripheral blood (Serra et al., 2017), and kidney cells (Aparicio-Soto et al., 2016; Incani et al., 2010; Deiana et al., 2011). It is noteworthy that the major metabolites of HT and Tyr, glucuronides and sulfates, which are usually found at higher concentrations than the parent compounds at systemic and intestinal level after olive products ingestion, showed in our experimental system to essentially retain parental biological activity, in accordance with previous data demonstrating the effectiveness of some 
glucuronide and sulfate metabolites in the regulation of many cell signals (Atzeri et al., 2016; Heleno et al., 2015).

Thus, it seems that glucuronide and sulfate chemical groups do not modify the properties of the parent compounds. This could be attributable to an eventual loss of the sulfate/glucuronide moiety once the metabolites enter Caco-2 cells so forming HT and Tyr, as previously demonstrated in the same experimental conditions (Atzeri et al., 2016). However, HT and Tyr themselves, once inside the cell, could be metabolized into sulfate and glucuronide forms, and act as metabolites, modulating cellular signaling pathways activated in response to oxidative stress and proinflammatory stimuli (Atzeri et al., 2016).

\subsection{Conclusions}

This study highlighted for the first time the ability of HT and Tyr sulfate and glucuronide metabolites to inhibit iNOS-derived NO overproduction in intestinal Caco- 2 cells. It should be emphasized that, as NO is one of the key mediators of the inflammatory process, this inhibitory action, that is likely to occur in the human intestinal lumen, may be particularly relevant in the maintenance of intestinal homeostasis. This effect was achieved through the modulation of IkB $\alpha$ degradation, due to inhibitory effects on MAPKs but not on Akt. Sulfates and glucuronides, major metabolites of HT and Tyr, showed an efficiency comparable to that of the parent compounds, strengthening the assumption that the potential beneficial effect of the ingested parent compounds may be retained, even when extensive metabolisation occurs.

The pool of compounds that concentrate at intestinal level following olive products ingestion, simple phenols and metabolites, all of which are probably in part responsible for the final effect, may significantly contribute to preserve intestinal integrity against pro-inflammatory agents, thus preventing or limiting the onset and progression of chronic intestinal inflammation and related diseases. 
Acknowledgments: We gratefully acknowledge FBS, Fondazione Banco di Sardegna (Prot.U915.2014/AI.798.MGB) and C.R.P. Regione Sardegna (L.R.7, Project "Sartol") for the financial support.

Conflict of Interest: The authors declared no conflicts of interest. 


\section{References}

Abbas, S., Alam, S., Pal, A., Kumar, M., Singh, D., \& Ansari, K. M. (2016). Food and Chemical Toxicology, 96, 183-190.

Aparicio-Soto, M., Sanchez-Fidalgo, S., Gonzalez-Benjumea, A., Maya, I., Fernandez-Bolaňos, J. G., \& Alarcon-de-la-Lastra, C. (2015). Naturally occurring hydroxytyrosol derivatives: hydroxytyrosyl acetate and 3,4-dihydroxyphenylglycol modulate inflammatory response in murine peritoneal macrophages. potential utility as new dietary supplements. Journal of Agricultural and Food Chemistry, 63, 836-846.

Aparicio-Soto, M., Sánchez-Hidalgo, M., Cárdeno, A., Rosillo, M. T., Sánchez-Fidalgo, S., Utrilla, J., Martín-Lacave, I., \& Alarcón-de-la-Lastra, C. (2016). Dietary extra virgin olive oil attenuates kidney injury in pristane-induced SLE model via activation of $\mathrm{HO}-1 / \mathrm{Nrf}-2$ antioxidant pathway and suppression of JAK/STAT, NF-кB and MAPK. Journal of Nutritional Biochemistry, 27, 278-288.

Atzeri, A., Lucas, R., Incani, A., Penalver, P., Zafra-Gomez, A., Melis, M. P., Pizzala, R., Morales, J. C., \& Deiana, M. (2016). Hydroxytyrosol and tyrosol sulfate metabolites protect against the oxidized cholesterol pro-oxidant effect in Caco-2 human enterocyte-like cells. Food and Function, 7(1), 337-46.

Bendini, A., Cerretani, L., Carrasco-Pancorbo, A., Gómez-Caravaca, A. M., Segura-Carretero, A., Fernández-Gutiérrez, A., \& Lercker, G. (2007). Phenolic Molecules in Virgin Olive Oils: a Survey of Their Sensory Properties, Health Effects, Antioxidant Activity and Analytical Methods. An Overview of the Last Decade. Molecules, 12, 1679-1719.

Bognar, E., Sarszegi, Z., Szabo, A., Debreceni, B., Kalman, N., Tucsek, S., Sumegi, B., \& Gallyas, F. Jr. (2013). Antioxidant and anti-inflammatory effects in Raw264.7 macrophages of malvidin, a major red wine polyphenol. Plos One, 8(6), e65355. 
Bradford, M.M.A., (1976). Rapid and sensitive method for the quantification of microgram quantities of protein utilizing the principle of protein-dye binding. Analytical Biochemistry, 72: $248-254$.

Bulotta, S., Celano, M., Lepore, S. M., Montalcini, T., Pujia, A., \& Russo, D. (2014). Beneficial effects of the olive oil phenolic components oleuropein and hydroxytyrosol: focus on protection against cardiovascular and metabolic diseases. Journal of Translational Medicine, $12,219$.

Cardeno, A., Sánchez-Hidalgo, M., Aparicio-Soto, M., Sánchez-Fidalgo, S., \& Alarcón-de-laLastra, C. (2014). Extra virgin olive oil polyphenolic extracts downregulate inflammatory responses in LPS-activated murine peritoneal macrophages suppressing NFאB and MAPK signalling pathways. Food and Function, 5(6), 1270-1277.

Casaburi, I., Puoci, F., Chimento, A., Sirianni, R., Ruggiero, C., Avena, P., \& Pezzi, V. (2013). Potential of olive oil phenols as chemopreventive and therapeutic agents against cancer: A review of in vitro studies. Molecular Nutrition and Food Research, 57, 71-83.

Catalan, U., López de las Hazas, M-C., Piñol C., Rubió, L., Motilva, M-J., Fernandez-Castillejo, S., Solà, R. (2018). Hydroxytyrosol and its main plasma circulating metabolites attenuate the initial steps of atherosclerosis through inhibition of the MAPK pathway. Journal of Functional Foods, 40, 280-291.

Catalan, U., López de las Hazas, M-C., Rubió, L., Fernandez-Castillejo, S., Pedret, A., de la Torre, R., Motilva, M-J., \& Solà, R. (2015). Protective effect of hydroxytyrosol and its predominant plasmatic human metabolites against endothelial dysfunction in human aortic endothelial cells. Molecular Nutrition and Food Research, 59, 2523-2536.

Chen, X. M., \& Kitts, D. D. (2015). Evidence for inhibition of nitric oxide and inducible nitric oxide synthase in Caco-2 and RAW 264.7 cells by a Maillard reaction product [5-(5,6- 
dihydro-4H-pyridin-3-ylidenemethyl)furan-2-yl]-methanol. Molecular and Cellular Biochemistry, 406, 205-215.

Chokshi, N. K., Hunter, C. J., Guner, Y. S., Grishin, A., \& Ford, H. R. (2008). The role of nitric oxide in intestinal epithelial injury and restitution in neonatal NEC. Seminars in Perinatology, 32(2), 92-99.

Cicerale, S., Lucas, L. J., \& Keast, R. S. J. (2012). Antimicrobial, antioxidant and antiinflammatory phenolic activities in extra virgin olive oil. Current Opinion in Biotechnology, $23,129-135$.

Corona, G., Spencer, J. P. E., \& Dessì, M. A. (2009). Extra virgin olive oil phenolics: absorption, metabolism, and biological activities in the GI tract. Toxicology and Industrial Health, 25, 285-293.

Cosan, D. T., Bayram, B., Soyocak, A., Basaran, A., Gunes, H. V., Degirmenci, I., \& Musmul, A. (2010). Role of phenolic compounds in nitric oxide synthase activity in colon and breast adenocarcinoma. Cancer Biotherapy and Radiopharmaceuticals, 25(5), 577-580.

De la Torre, R. (2008). Bioavailability of olive oil phenolic compounds in humans. Inflammopharmacology, 16, 245-247.

Deiana, M., Serra, G., \& Corona, G. (2018). Modulation of intestinal epithelium homeostasis by extra virgin olive oil phenolic compounds. Food and Function, 9, 4085-4099.

Deiana, M., Incani, A., Rosa, A., Atzeri, A., Loru, D., Cabboi, B., Melis, M. P., Lucas, R., Morales, J. C., \& Dessì, M. A. (2011). Hydroxytyrosol glucuronides protect renal tubular epithelial cells against H2O2 induced oxidative damage. Chemico-Biological Interactions, 193, 232239. 
Förstermann, U., \& Sessa, W. C. (2012). Nitric oxide synthases: regulation and function. European Heart Journal, 33, 829-837.

Fu, P., \& Hu, Q. (2016). 3,4-Dihydroxyphenylethanol alleviates early brain injury by modulating oxidative stress and Akt and nuclear factor- $\kappa \mathrm{B}$ pathways in a rat model of subarachnoid hemorrhage. Experimental and Therapeutic Medicine, 11, 1999-2004.

Guichard, C., Pedruzzi, E., Fay, M., Marie, J. C., Braut-Boucher, F., Daniel, F., Grodet, A., Gougerot-Pocidalo, M. A., Chastre, E., Kotelevets, L., Lizard, G., Vandewalle, A., Driss, F., \& Ogier-Denis E. (2006). Dihydroxyphenylethanol induces apoptosis by activating serine/threonine protein phosphatase $\mathrm{PP} 2 \mathrm{~A}$ and promotes the endoplasmic reticulum stress response in human colon carcinoma cells. Carcinogenesis, 27, 1812-1827.

Guo, S., Al-Sadi, R., Said, H. M., \& Ma, T. Y. (2013). Lipopolysaccharide causes an increase in intestinal tight junction permeability in vitro and in vivo by inducing enterocyte membrane expression and localization of TLR-4 and CD14. American Journal of Pathology, 182, 375387.

Guo, S., Nighot, M., Al-Sadi, R., Alhmoud, T., Nighot, P., \& Ma, T. Y. (2015). Lipopolysaccharide regulation of intestinal tight junction permeability is mediated by TLR4 signal transduction pathway activation of FAK and MyD88. Journal of Immunology, 195(10), 4999-5010.

Gupta, S. C., Sundaram, C., Reuter, S., \& Aggarwal, B. B. (2010). Inhibiting NF-кB activation by small molecules as a therapeutic strategy. Biochimica et Biophysica Acta, 1799(10-12), 775787.

Habib, S., \& Ali, A. (2011). Biochemistry of Nitric Oxide. Indian Journal of Clinical Biochemistry, 26(1), 3-17. 
He, H., Feng, Y. S., Zang, L. H., Liu, W. W., Ding, L. Q., Chen, L. X., Kang, N., Hayashi, T., Tashiro, S., Onodera, S., Qiu, F., \& Ikejima, T. (2014). Nitric oxide induces apoptosis and autophagy; autophagy down-regulates NO synthesis in physalin A-treated A375-S2 human melanoma cells. Food and Chemical Toxicology, 71, 128-135.

Heleno, S. A., Martins, A., Queiroz, M. J. R. P., \& Ferreira, I. C. F. R. (2015). Bioactivity of phenolic acids: Metabolites versus parent compounds: A review. Food Chemistry, 173(15), $501-513$

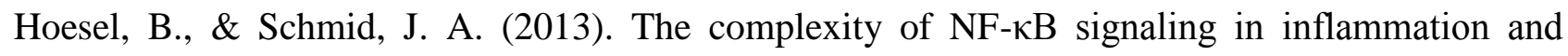
cancer. Molecular Cancer, 12:86.

Hu, T., He, X-W., Jiang, J-G., \& Xu, X-L. (2014). Hydroxytyrosol and its potential therapeutic effects. Journal of Agricultural and Food Chemistry, 62, 1449-1455.

Incani, A., Deiana, M., Corona, G., Vafeiadou, K., Vauzour, D., Dessì, M. A., \& Spencer, J. P. (2010). Involvement of ERK, Akt and JNK signalling in H2O2-induced cell injury and protection by hydroxytyrosol and its metabolite homovanillic alcohol. Molecular Nutrition and Food Research, 54(6), 788-796.

Incani, A., Serra, G., Atzeri, A., Melis, M. P., Serreli, G., Bandino, G., Sedda, P., Campus, M., Tuberoso, C. I. G., \& Deiana, M. (2016). Extra virgin olive oil phenolic extracts counteract the pro-oxidant effect of dietary oxidized lipids in human intestinal cells. Food and Chemical Toxicology, 90, 171-180.

Jenner, A. M., Rafter, J., \& Halliwell, B. (2005). Human fecal water content of phenolics: The extent of colonic exposure to aromatic compounds. Free Radical Biology \& Medicine, 38, 763-772. 
Jochems, P. G. M., Garssen, J., van Keulen, A. M., Masereeuw, R., \& Jeurink, P. V. (2018). Evaluating human intestinal cell lines for studying dietary protein absorption. Nutrients, 10(3), 322 .

Kanarek, N., London, N., Schueler-Furman, O., \& Ben-Neriah, Y. (2010). Ubiquitination and degradation of the inhibitors of NF-kappaB. Cold Spring Harbor Perspectives in Biology, 2(2), a000166.

Kane, L. P., Shapiro, V. S., Stokoe, D., \& Weiss, A. (1999). Induction of NF-kappaB by the Akt/PKB kinase. Current Biology, 9(11), 601-604.

Kleinert, H., Schwarz, P. M., \& Forstermann, U. (2003). Regulation of the expression of inducible Nitric Oxide Synthase. Biological Chemistry, 384, 1343-1364.

Maiuri, M. C., De Stefano, D., Di Meglio, P., Irace, C., Savarese, M., Sacchi, R., Cinelli, M. P., \& Carnuccio, R. (2005). Hydroxytyrosol, a phenolic compound from virgin olive oil, prevents macrophage activation. Naunyn-Schmiedeberg's Archives of Pharmacology, 371(6), 457-465.

Marion, R., Coeffier, M., Leplingard, A., Favennec, L., Ducrotte, P., \& Dechelotte, P. (2003). Cytokine-stimulated nitric oxide production and inducible NO-synthase mRNA level in human intestinal cells: lack of modulation by glutamine. Clinical Nutrition, 22, 523-528.

Miro-Casas, E., Covas, M. I., Farre, M., Fito, M., Ortuno, J., Weinbrenner, T., Roset, P., \& de la Torre, R. (2003). Hydroxytyrosol disposition in humans. Clinical Chemistry, 49(6), 945-952.

Murakami, A. (2009). Chemoprevention with phytochemicals targeting inducible nitric oxide synthase. Forum of Nutrition, 61, 193-203.

Muriana, F. J. G., Montserrat-de la Paz, S., Lucas, R., Bermudez, B., Jaramillo, S., Morales, J. C., Abia, R., \& Lopez, S. (2017). Tyrosol and its metabolites as antioxidative and antiinflammatory molecules in human endothelial cells. Food and Function, 8(8), 2905-2914. 
Panaro, M. A., Carofiglio, V., Acquafredda, A., Cavallo, P., \& Cianciulli, A. (2012). Antiinflammatory effects of resveratrol occur via inhibition of lipopolysaccharide-induced NF-kB activation in Caco-2 and SW480 human colon cancer cells. British Journal of Nutrition, 108, $1623-1632$.

Pastor, A., Rodrıguez-Morato, J., Olesti, E., Pujadas, M., Pérez-Mañá, C., Khymenets, O., Fitó, M., Covas, M. I., Solá, R., Motilva, M. J., Farré, M., \& de la Torre, R. (2016). Analysis of free hydroxytyrosol in human plasma following the administration of olive oil. Journal of Chromatography A, 1437, 183-190.

Rao, C. V. (2004). Nitric oxide signaling in colon cancer chemoprevention. Mutation Research, $555,107-119$.

Rodriguez-Morato, J., Boronat, A., Kotronoulas, A., Pujadas, M., Pastor, A., Olesti, E., PérezMañá, C., Khymenets. O., Fitó, M., Farré, M., \& De la Torre, R. (2016). Metabolic disposition and biological significance of simple phenols of dietary origin: hydroxytyrosol and tyrosol. Drug Metabolism Reviews, 48(2), 218-236.

Romier, B., Van De Walle, J., During, A., Larondelle, Y., \& Schneider, Y-J. (2008). Modulation of signalling nuclear factor-kB activation pathway by polyphenols in human intestinal Caco-2 cells. British Journal of Nutrition, 100, 542-551.

Romier-Crouzet, B., Van de Walle, J., During, A., Joly, A., Rousseau, C., Henry, O., Larondelle, Y., \& Schneider, Y. J. (2009). Inhibition of inflammatory mediators by polyphenolic plant extracts in human intestinal Caco-2 cells. Food and Chemical Toxicology, 47, 1221-1230.

Rosa, A., Atzeri, A., Deiana, M., Melis, M. P., Loru, D., Incani, A., Cabboi, B., \& Dessì, M. A. (2011). Effect of aqueous and lipophilic mullet (Mugil cephalus) Bottarga extracts on the growth and lipid profile of intestinal Caco-2 cells. Journal of Agricultural and Food Chemistry, 59(5), 1658-1666. 
Rubió, L., Macia, A., Castell-Auvi, A., Pinent, M., Blay, M. T., Ardevol, A., Romero, M. P., \& Motilva M. J. (2014a). Effect of the co-occurring olive oil and thyme extracts on the phenolic bioaccesibility and bioavailability assessed by in vitro digestion and cell models. Food Chemistry, 149, 277-284.

Rubió, L., Serra, A., Macià, A., Piñol, C., Romero, M. P., \& Motilva, M. J. (2014b). In vivo distribution and deconjugation of hydroxytyrosol phase II metabolites in red blood cells: A potential new target for hydroxytyrosol. Journal of Functional Foods, 10, 139-143.

Santangelo, C., Varì, R., Scazzocchio, B., de Sanctis, P., Giovannini, C., D'Archivio, M., \& Masella, R. (2018). Anti-inflammatory Activity of Extra Virgin Olive Oil Polyphenols: Which Role in the Prevention and Treatment of Immune-Mediated Inflammatory Diseases? Endocrine, Metabolic and Immune Disorders - Drug Targets, 18(1), 36-50.

Scalbert, A., \& Williamson, G. (2000). Dietary intake and bioavailability of polyphenols. Journal of Nutrition, 130, 2073-2085.

Serra, G., Deiana, M., Spencer, J. P. E., \& Corona, G. (2017). Olive oil phenolics prevent oxysterolinduced proinflammatory cytokine secretion and reactive oxygen species production in human peripheral blood mononuclear cells, through modulation of p38 and JNK pathways. Molecular Nutrition and Food Research, 61, 1-7.

Serra, G., Incani, A., Serreli, G., Porru, L., Melis, M. P., Tuberoso, C. I. G., Rossin, D., Biasi, F., \& Deiana, M. (2018). Olive oil polyphenols reduce oxysterols -induced redox imbalance and pro-inflammatory response in intestinal cells. Redox Biology, 17, 348-354.

Serreli, G., \& Deiana, M. (2018). Biological relevance of extra virgin olive oil polyphenols metabolites. Antioxidants, 7(170), 1-17. 
Serreli, G., Incani, A., Atzeri, A., Angioni, A., Campus, M., Cauli, E., Zurru, R., \& Deiana, M. (2017). Antioxidant effect of natural table olives phenolic extract against oxidative stress and membrane damage in enterocyte-like cells. Journal of Food Science, 82(2), 380-385.

Somchit, M., Changtam, C., Kimseng, R., Utaipam, T., Lertcanawanichakul, M., Suksamrarn, A., \& Chunglok, W. (2014). Demethoxycurcumin from Curcuma longa rhizome suppresses iNOS induction in an in vitro inflamed human intestinal mucosa model. Asian Pacific Journal of Cancer Prevention, 15(4), 1807-1810.

Soufli, I., Toumi, R., Rafa, H., \& Touil-Boukoffa, C. (2016). Overview of cytokines and nitric oxide involvement in immuno-pathogenesis of inflammatory bowel diseases. World Journal of Gastrointestinal Pharmacology and Therapeutics, 6, 7(3), 353-360.

Sun, P., Zhou, K., Wang, S., Li, P., Chen, S., Lin, G., Zhao, Y., \& Wang, T. (2013). Involvement of MAPK/NF- $\kappa \mathrm{B}$ signaling in the activation of the cholinergic anti-inflammatory pathway in experimental colitis by chronic vagus nerve stimulation. PLoS One, 8, e69424.

Van De Walle, J., Hendrickx, A., Romier, B., Larondelle, Y., \& Schneider, Y-J. (2010). Inflammatory parameters in Caco-2 cells: Effect of stimuli nature, concentration, combination and cell differentiation. Toxicology in Vitro, 24, 1441-1449.

Viatour, P., Merville, M-P. Bours, V., \& Chariot A., (2005). Phosphorylation of NF-kB and IkB proteins: implications in cancer and inflammation. Trends in Biochemical Sciences, 30(1), 4352.

Xiong, Y., Chen, D., Yu, C., Lv, B., Peng, J., Wang, J., \& Lin, Y. (2015). Citrus nobiletin ameliorates experimental colitis by reducing inflammation and restoring impaired intestinal barrier function. Molecular Nutrition and Food Research, 59, 829-842. 
Zhao, B., Ma, Y., Xu, Z., Wang, J., Wang, F., Wang, D., Pan, S., Wu, Y., Pan, H., Xu, D., Liu, L., \& Jiang, H. (2014). Hydroxytyrosol, a natural molecule from olive oil, suppresses the growth of human hepatocellular carcinoma cells via inactivating AKT and nuclear factor-kappa B pathways. Cancer Letters, 347, 79-87. 